\title{
EFFECT OF ORAL AZITHROMYCIN AND METRONIDAZOLE AS AN ADJUNCT TO SCALING AND ROOT PLANING ON GLYCEMIC CONTROL IN TYPE II DIABETIC PATIENTS WITH CHRONIC PERIODONTITIS
}

\author{
DHIVYA K ${ }^{1 *}$, YOGARAJAN K ${ }^{2}$, SHANMUGARAJAN TS ${ }^{1}$ \\ ${ }^{1}$ Department of Pharmacy Practice and Pharm D, School of Pharmaceutical Sciences, Vels University (VISTAS), Chennai, Tamil Nadu, \\ India. ${ }^{2}$ Department of Periodontology, Sri Danwanthri Dental Care, Vellore, Tamil Nadu, India. Email: divyapharmd@gmail.com
}

Received: 07 December 2016, Revised and Accepted: 24 December 2016

\section{ABSTRACT}

Objective: Periodontitis, a chronic inflammatory disease characterized by destruction of the periodontal ligament and alveolar bone is the sixth complication of diabetes mellitus. Periodontal treatment that reduces gingival inflammation aids in the control of hyperglycemia. Therefore, the present study was designed to determine the effect of treating chronic periodontitis with oral antibiotics azithromycin and metronidazole on the level of serum glycated hemoglobin in type-II diabetic patients.

Methods: This prospective observational study was carried out in the dental department of a tertiary care hospital for 9 months. Clinical and biochemistry reports of 90 patients were collected in designed case report forms. All statistical analyses were performed using IBM Statistical Package for Social Sciences 17 and Graph Pad Prism 7.0.

Results: Significant reduction in all the clinical and dental parameters was comparatively higher in patients who received azithromycin than in patients who received metronidazole and scaling and root planning alone.

Conclusion: Periodontal therapy with oral azithromycin can be employed as a supportive strategy for the management of diabetes mellitus. Henceforth, prevention and control of periodontal disease along with antibiotics must be considered an integral part of glycemic control. However, due to the lesser sample size in this study, further investigations are required to confirm the effect of periodontal therapy on systemic diseases.

Keywords: Periodontitis, Azithromycin, Metronidazole, Glycemic control, Diabetes mellitus.

(c) 2017 The Authors. Published by Innovare Academic Sciences Pvt Ltd. This is an open access article under the CC BY license (http://creativecommons. org/licenses/by/4. 0/) DOI: http://dx.doi.org/10.22159/ajpcr.2017.v10i3.16500

\section{INTRODUCTION}

Oral hygiene plays a critical role in whole-body health. There has been an emerging interest in the interrelationship between systemic conditions and oral health. Periodontal diseases are a group of inflammatory, microbial-induced infections involving the supporting tissues of the teeth and mainly caused by gram-negative, anaerobic bacteria $[1,2]$. The American Society of Diabetes defined periodontal disease as the sixth complication of diabetes in 1997 [3]. Pathogens which are commonly involved in causing periodontitis are Porphyromonas gingivalis, Prevotella intermedia, Tannarella forsythia and Aggregatibacter actinomycetmcomintans, which lead to soft and hard tissue destruction, dental mobility, and the loss of dental elements [4,5]. The inflamed periodontal tissue may serve as a chronic source of bacteria, bacterial products and may trigger the release of inflammatory mediators such as tumor necrosis factor- $\alpha$ (TNF- $\alpha$ ), interleukin (IL) 6 and 1 which have important effects on lipid and glucose metabolism [6]. DM is a metabolic disorder characterized by hyperglycemia resulting from a defective insulin secretion and/or activity. Periodontitis is an inflammatory response to a bacterial challenge and represents a portal of entry for periodontal pathogens, bacterial endotoxins, and proinflammatory cytokines [7]. Inflammation is a central feature of the pathogenesis of diabetes and periodontitis. TNF- $\alpha$ and IL- 6 are the main inducers of acute-phase proteins, including C-reactive protein (CRP), and both have been shown to impair intracellular insulin signaling, potentially contributing to insulin resistance [8]. The link between diabetes and periodontitis is bidirectional. Diabetes has well been established as a major risk factor for periodontitis and direct relationships have been demonstrated between degree of hyperglycemia and severity of periodontitis. Similarly, mechanisms underlying the effect of periodontal inflammation on blood glucose level have also been proven [9]. The presence of periodontal diseases can have a significant impact on the metabolic state in diabetes. Periodontitis is also associated with an increased risk for diabetic complications. Studies have shown that diabetic patients with periodontal infection have a greater risk of worsening glycemic control over time compared to diabetic subjects without periodontitis [10]. Thus, the local oral inflammatory disease, periodontitis, may induce and perpetuate a systemic inflammation that may aggravate systemic diseases such as cardiovascular disease, pulmonary disease, rheumatoid arthritis, and diabetes mellitus [7]. Periodontal treatment that reduces gingival inflammation aids in the control of hyperglycemia. Antibiotics are important adjuncts in the treatment of infectious diseases, including periodontitis [11]. Among the various antibiotics, azithromycin, and metronidazole are selected for the study. Azithromycin is a macrolide, broad spectrum antibiotic whose concentration in gingival crevicular fluid (GCF) is higher than that of serum and also exerts post antibiotic effect. In addition, azithromycin is preferentially taken up by phagocytes, and hence, its level in infected tissues is much higher than in similar noninfected sites. Azithromycin has been used for the treatment of chronic periodontitis [12-14]. Metronidazole is a nitroimidazole bactericidal antibiotic and effective against anaerobes. Oral metronidazole is widely used in chronic periodontitis treatment due to its anti-anaerobic effect [15]. Metronidazole can readily attain effective anti-bacterial concentrations in gingival tissue and crevicular fluid [16]. Hence, this study is designed to assess the effect of periodontal therapy on glycated hemoglobin (HbA1c) in patients with type 2 diabetes mellitus by treating the chronic periodontitis using azithromycin and metronidazole following scaling and root planning (SRP). 


\section{METHODS}

The study entitled "effect of azithromycin and metronidazole for chronic periodontitis on glycemic control in type II diabetes" was conducted as a prospective observational study in the dental department of Tertiary Care Hospital for 9 months. The study protocol was approved by the institutional ethics committee of Vels University (Approval no: IEC/ DOPI/2015/21). The schedule for patient follow-up and treatment plan is shown in Table 1.

\section{Consent from hospital authority}

The protocol of the proposed study was submitted as printed copies to the medical superintendent and dentists for review and approval. Guidance of the senior dentist available was extended on request from the scholar. Consent from the hospital authorities and dentists were obtained before accessing the data from the patients. Clinical and biochemistry data of the patients who fulfilled the inclusion criterion were documented from the case sheets and recorded in a separately designed case report form.

\section{Study design}

The study comprised three groups and each group included 30 patients as follows:

- Group I: 30 patients treated with SRP and azithromycin (500 mg OD $\times 3$ days)

- Group II: 30 patients treated with SRP and metronidazole (250 mg TDS $\times 5$ days)

- Group III: 30 patients treated with SRP.

SRP was done using ultrasonic scaler and Gracey's curettes.

\section{Patient recruitment}

Detailed information regarding disease condition, treatment plan, and oral hygiene instructions were provided to the patients willing to participate in the study. Written informed consents were obtained after explaining the study protocol to each individual patient.

\section{Inclusion criteria}

Patients of both gender between 35 and 70 years of age with uncontrolled type II DM and chronic periodontitis that has a probing depth of $4 \mathrm{~mm}-7 \mathrm{~mm}$ in at least 3 sites were included in the study.

\section{Exclusion criteria}

Patients on regular anti-inflammatory medications, who have taken systemic antibiotics within the last 3 months before enrollment, patients with significant history of smoking, tobacco use, alcoholism and drug abuse, pregnant and lactating women, patients who have received periodontal therapy within the last 6 months, patients with less than 8 natural teeth in oral cavity and poor dental prognosis, patients who are allergic to azithromycin and metronidazole and patients with major medical complications were excluded from the study.

\section{Clinical parameters}

1. Gingival index - Loe and Silness [17]

2. Plaque index - Loe and Silness [17].

3. Pocket probing depth was recorded to nearest millimeter using UNC 15 probe [18].

4. Clinical attachment levels in millimeter from reference point (cemento enamel junction) to the base of pocket using customized occlusal acrylic stents and UNC 15 probe [18].

5. HbA1c, serum CRP and lipid profile - collected from case sheets.

\section{Statistical analysis}

Comparison between three groups was analyzed by means of oneway analysis of variance (ANOVA) and student $t$-test to determine the presence or absence of statistically significant difference wherever necessary. Wherever computed, the value of $\mathrm{p}<0.05$ was considered statistically significant, since the confidence interval was maintained at 95\%. Univariate analyses were carried out by Pearson's correlation. Predictors of reduction in $\mathrm{HbA1c}$ level was determined by multiple linear regression analysis. All statistical analyses were performed using IBM Statistical Package for Social Sciences 17 and Graph Pad Prism 7.0.

\section{RESULTS}

\section{Patient demographics}

A total of 90 patients who fulfilled the inclusion criteria were enrolled in the study. The demographic and clinical characteristics of the patients included in the study are shown in Tables 2 and 3.

The mean \pm standard deviation $\left(\mathrm{SD}_{-}\right.$age of the studied population was found to be 51.2 (7.51) years with a median age of 52 years. 52 (57.7\%) patients were male, whereas $38(42.2 \%)$ patients were female. Statistically significant difference was not observed between mean \pm SD age of males (50.92 [8.01]) and females (51.57 [6.74]) ( $p=0.6865)$.

Statistically significant difference in the above parameters was not found between genders (unpaired $t$-test).

\section{Effect of oral azithromycin and metronidazole on HbA1c level}

The presence of statistically significant difference in HbA1c between baseline and third month was (intra group comparison) determined by means of student $t$-test, whereas one-way ANOVA was used to determine inter group difference. A mean decrease in HbA1c level was observed during the $3^{\text {rd }}$ month. Inter group comparison through one way ANOVA showed periodontitis treatment with oral azithromycin as an adjunct to SRP to be more effective on glycemic control when compared to the other two groups as shown in Table 4. Changes in HbA1c levels after 3 months of periodontal therapy was found to be significantly high in azithromycin treated group than metronidazole treated group as shown in Fig. 1.

Determination of factors influencing change in HbA1c $(\triangle \mathrm{HbA1c})$ : Effect of periodontal therapy

Logistic linear regression analysis was carried out to determine factors that cause change in HbA1c levels. Expected explanatory variables were regressed against the dependent variable, $\Delta \mathrm{HbA} 1 \mathrm{c}$. Concomitant insulin therapy, change in CRP, life style modification and periodontal therapy were found to be significant covariates affecting $\triangle$ HbA1c. Thus, periodontal therapy was found to be a significant factor in achieving glycemic control in type II diabetes patients with chronic periodontitis. The results of the regression analysis are shown in Table $5 \mathrm{a}$ and $\mathrm{b}$.

Effect of oral azithromycin and metronidazole on dental parameters

Gingival index, plaque index, probing depth, and clinical attachment loss were observed to be significantly decreased after periodontal therapy in chronic periodontitis patients. Oral azithromycin therapy as an adjunct to SRP was found to be comparatively more effective in treating chronic periodontitis than oral metronidazole and SRP alone groups as shown in Tables 6-9.

Table 1: Review schedule

\begin{tabular}{llll}
\hline Review & Group I (30 patients) & Group II (30 patients) & Group III (30 patients) \\
\hline R0 (baseline) & Before periodontitis treatment & Before periodontitis treatment & Before periodontitis treatment \\
R1 (1 month) & SRP+azithromycin & SRP+metronidazole & SRP \\
R2 (3 months) & SRP+azithromycin & SRP+metronidazole & SRP \\
\hline
\end{tabular}

R0=Baseline review, R1=Review 1, R2=Review 2, OHI: Oral hygiene instructions, SRP: Scaling and root planning 


\section{DISCUSSION}

The effect of periodontal inflammation on systemic and metabolic diseases has been well established. The current therapeutic regimens for periodontitis include the use of local and systemic antibiotics with scaling, root planning, and oral hygiene practices [19]. Local antibiotics used are tetracycline fibers, minocycline gel, etc.

Table 2: Age-wise distribution

\begin{tabular}{llll}
\hline $\begin{array}{l}\text { Age } \\
\text { range }\end{array}$ & $\begin{array}{l}\text { Number of patients (\%) } \\
\mathbf{n = 9 0}\end{array}$ & Mean \pm SD & Age quartile \\
\hline $31-40$ & $9(10)$ & $37.88 \pm 1.52$ & $35-40$ \\
$41-50$ & $31(34.4)$ & $46.03 \pm 2.45$ & $41-50$ \\
$51-60$ & $40(44.4)$ & $55.17 \pm 2.59$ & $51-60$ \\
$61-70$ & $10(11.11)$ & $63.4 \pm 1.49$ & $62-66$ \\
\hline
\end{tabular}

SD: Standard deviation

Table 3: Clinical and periodontal characteristics of studied patients

\begin{tabular}{lllll}
\hline Parameter & Mean \pm SD & Median & Range & p \\
\hline BMI $\left(\mathrm{kg} / \mathrm{m}^{2}\right)$ & $29.6 \pm 2.52$ & 29 & $19.4-36.5$ & 0.096 \\
Plaque index & $2.32 \pm 0.44$ & 2.4 & $1.2-3$ & 0.5207 \\
Gingival index & $2.15 \pm 0.46$ & 2.35 & $1.2-2.8$ & 0.9380 \\
PPD (mm) & $5.32 \pm 0.92$ & 5 & $8-15$ & 0.1901 \\
CAL $(\mathrm{mm})$ & $11.01 \pm 1.78$ & 11 & $8-15$ & 0.1070 \\
HbA1c $(\%)$ & $11.36 \pm 1.47$ & 11.35 & $8.8-14.8$ & 0.3659 \\
\hline
\end{tabular}

BMI: Body mass index, SD: Standard deviation, HbA1c: Glycated hemoglobin

Table 4: Comparison of baseline and post-treatment HbA1c level

\begin{tabular}{llll}
\hline Group & HbA1c & \\
\cline { 2 - 4 } & Baseline & 3 months & p $^{*}$ \\
\hline I $(\mathrm{n}=30)$ & $11.46 \pm 1.57$ & $9.28 \pm 0.81$ & 0.000 \\
II $(\mathrm{n}=30)$ & $11.38 \pm 1.47$ & $10.47 \pm 1.45$ & 0.000 \\
III $(\mathrm{n}=30)$ & $11.25 \pm 1.38$ & $11.20 \pm 1.34$ & 0.033 \\
$\mathrm{p}^{* *}$ & 0.352 & 0.0332 & \\
\hline
\end{tabular}

${ }^{*} \mathrm{p}$ value retrieved by paired student $t$-test, ${ }^{* *} \mathrm{p}$ value retrieved by one-way

ANOVA, HbA1c: Glycated hemoglobin, ANOVA: Analysis of variance

Table 5a: Model summary

\begin{tabular}{lllll}
\hline Model & $\mathbf{R}$ & $\mathbf{R}^{2}$ & Adjusted $\mathbf{R}^{2}$ & $\begin{array}{l}\text { Standard error of the } \\
\text { estimate }\end{array}$ \\
\hline 1 & $0.768^{\mathrm{a}}$ & 0.590 & 0.585 & 0.79279 \\
2 & $0.826^{\mathrm{b}}$ & 0.682 & 0.675 & 0.70157 \\
3 & $0.839^{\mathrm{c}}$ & 0.704 & 0.694 & 0.68068 \\
4 & $0.855^{\mathrm{d}}$ & 0.731 & 0.718 & 0.65369 \\
\hline
\end{tabular}

aPredictors: (Constant), insulin therapy, ${ }^{\mathrm{b} P r e d i c t o r s: ~(C o n s t a n t), ~ i n s u l i n ~ t h e r a p y, ~}$ change in CRP, 'Predictors: (Constant), insulin therapy, change in CRP, life style modification, ${ }^{\mathrm{d} P r e d i c t o r s: ~(C o n s t a n t), ~ i n s u l i n ~ t h e r a p y, ~ c h a n g e ~ i n ~ C R P, ~ l i f e ~ s t y l e ~}$ modification, periodontal treatment, CRP: C-reactive protein, CRP: C-reactive protein whereas systemic antibiotic therapy comprises the use of antibiotics such as amoxicillin, macrolides, clindamycin, fluoroquinolones, cephalosporins, and metronidazole [20]. In the current study, effectiveness of azithromycin and metronidazole has been comparatively evaluated. Longer half-life $\left(t_{1 / 2}\right)$ of azithromycin aids increased dosing interval and decreased the course of treatment. Further azithromycin exerts post antibiotic effect conferring longer protection to oral cavity [21]. On the other hand, metronidazole is widely used in periodontal therapy since organisms associated with periodontal infection are often gram negative anaerobes. In the current study, the effectiveness of azithromycin and metronidazole was comparatively assessed in uncontrolled diabetic patients who underwent scaling and root planning. Patients who received azithromycin showed statistically significant reduction in gingival index, plaque index, pocket probing depth, and clinical attachment loss at first and third month post therapy. The decrease in intensity of periodontal infection was comparatively higher in patients who received azithromycin than in patients who received metronidazole and SRP alone as shown in Tables 6-9. As discussed earlier, the potential efficacy of azithromycin could be attributed to its ability to attain higher concentrations in the GCF making it an ideal treatment choice for chronic periodontitis [22].

Chronic periodontitis has negative impact on glycemic control. Local production of cytokines in the periodontal tissue affects glycemic control through systemic exposure and impairing intracellular insulin signaling thus potentially contributing to insulin resistance [23]. Hyperglycemic state may thus be enhanced in chronic periodontitis patients and periodontal therapy can therefore result in reduced $\mathrm{HbA} 1 \mathrm{c}$ [24]. In this study, patients who received azithromycin showed comparatively higher reduction in $\mathrm{HbA1c}$ than patients who received metronidazole and SRP alone. The mean \pm SD reduction in HbA1c after 3 months was 2.18 (1.46) for azithromycin treated patients, whereas it was $1.09(0.46)$ and $0.14(0.24)$ for metronidazole and SRP alone group, respectively. Statistically significant difference in mean $\mathrm{HbA1c}$ reduction was found between

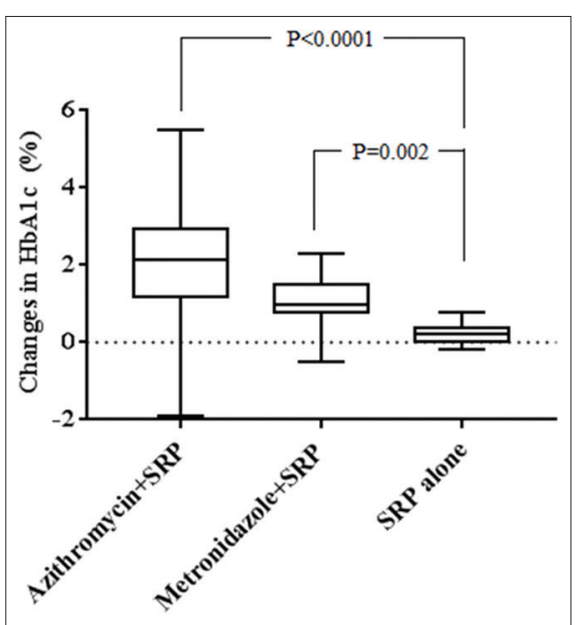

Fig. 1: Effect of oral antibiotic therapy on HbA1c level

Table 5b: Coefficients

\begin{tabular}{|c|c|c|c|c|c|}
\hline \multirow[t]{2}{*}{ Model } & \multicolumn{2}{|c|}{ Unstandardized coefficients } & \multirow{2}{*}{$\begin{array}{l}\text { Standardized coefficients } \\
\text { Beta }\end{array}$} & \multirow[t]{2}{*}{$\mathbf{t}$} & \multirow[t]{2}{*}{ Significant } \\
\hline & Beta & Standard error & & & \\
\hline \multicolumn{6}{|l|}{4} \\
\hline Insulin therapy & 1.023 & 0.221 & 0.379 & 4.637 & 0.000 \\
\hline Change in CRP & 1.233 & 0.413 & 0.264 & 2.988 & 0.004 \\
\hline Lifestyle & 0.627 & 0.217 & 0.186 & 2.892 & 0.005 \\
\hline Periodontal treatment & 0.358 & 0.125 & 0.227 & 2.872 & 0.005 \\
\hline
\end{tabular}

Dependent variable: Change in HbA1c, CRP: C-reactive protein, HbA1c: Glycated hemoglobin 
Table 6: Comparison of baseline and post-treatment gingival index

\begin{tabular}{lllll}
\hline Group & \multicolumn{2}{l}{ Gingival index } & \\
\cline { 2 - 5 } & Baseline & 1 month & 3 months & p \\
\hline I $(\mathrm{n}=30)$ & $2.20 \pm 0.49$ & $1.51 \pm 0.47$ & $1.29 \pm 0.46$ & \\
II $(\mathrm{n}=30)$ & $2.10 \pm 0.43$ & $1.77 \pm 0.44$ & $1.52 \pm 0.41$ & 0 \\
III $(\mathrm{n}=30)$ & $2.16 \pm 0.48$ & $1.95 \pm 0.45$ & $1.65 \pm 0.46$ & 0.001 \\
$\mathrm{p}$ & 0.72 & 0.013 & 0.047 & 0 \\
\hline
\end{tabular}

Table 7: Comparison of baseline and post-treatment plaque index

\begin{tabular}{lllll}
\hline \multirow{2}{*}{ Group } & \multicolumn{3}{l}{ Plaque index } & \\
\cline { 2 - 5 } & Baseline & 1 month & 3 months & p \\
\hline I $(\mathrm{n}=30)$ & $2.38 \pm 0.45$ & $1.54 \pm 0.33$ & $1.30 \pm 0.32$ & 0.000 \\
II $(\mathrm{n}=30)$ & $2.30 \pm 0.44$ & $1.83 \pm 0.41$ & $1.48 \pm 0.36$ & 0.000 \\
III $(\mathrm{n}=30)$ & $2.28 \pm 0.43$ & $1.96 \pm 0.44$ & $1.58 \pm 0.41$ & 0.000 \\
$\mathrm{p}$ & 0.648 & 0.000 & 0.013 & \\
\hline
\end{tabular}

Table 8: Comparison of baseline and post-treatment pocket probing depth

\begin{tabular}{lllll}
\hline \multirow{2}{*}{ Group } & \multicolumn{2}{l}{ Probing depth } & \\
\cline { 2 - 5 } & Baseline & 1 month & 3 months & p \\
\hline I $(\mathrm{n}=30)$ & $5.43 \pm 0.99$ & $4.63 \pm 0.80$ & $4.13 \pm 0.50$ & 0.000 \\
II $(\mathrm{n}=30)$ & $5.23 \pm 0.92$ & $4.70 \pm 0.74$ & $4.40 \pm 0.55$ & 0.000 \\
III $(\mathrm{n}=30)$ & $5.30 \pm 0.86$ & $5.03 \pm 0.66$ & $4.80 \pm 0.60$ & 0.033 \\
$\mathrm{p}$ & 0.704 & 0.089 & 0.075 & \\
\hline
\end{tabular}

Table 9: Comparison of baseline and post-treatment clinical attachment loss

\begin{tabular}{lllll}
\hline \multirow{2}{*}{ Group } & \multicolumn{4}{l}{ Clinical attachment loss } \\
& Baseline & 1 month & 3 months & p \\
\hline I $(n=30)$ & $11.07 \pm 2.00$ & $9.07 \pm 1.95$ & $8.33 \pm 1.87$ & 0.000 \\
II $(n=30)$ & $11.00 \pm 1.81$ & $9.90 \pm 1.35$ & $9.07 \pm 1.39$ & 0.000 \\
III $(n=30)$ & $10.97 \pm 1.52$ & $10.30 \pm 1.16$ & $9.53 \pm 1.09$ & 0.000 \\
p & 0.976 & 0.009 & 0.010 & \\
\hline
\end{tabular}

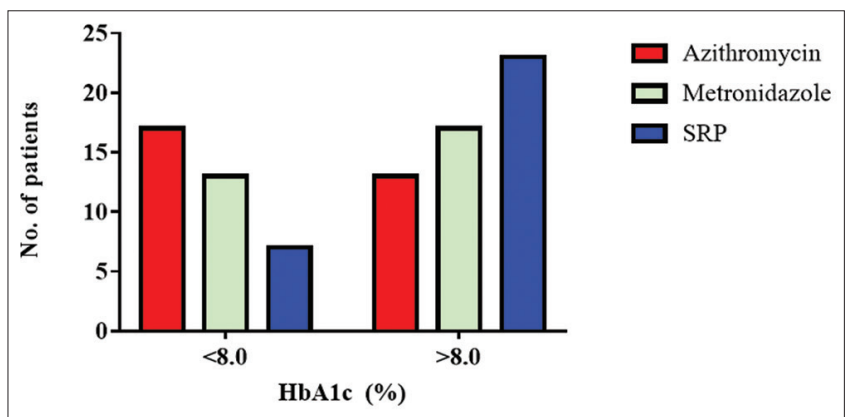

Fig. 2: Incidence of glycemic control between treatment groups. Statistically significant difference in incidence of diabetic control was found between azithromycin, metronidazole and scaling and root planning alone groups $(p=0.0306)$

patients who received concomitant antibiotic therapy and patients who received SRP alone as shown in Fig. 1. Similarly, the incidence of diabetic control was found to be high in azithromycin treated patients suggestive of a potential role of azithromycin in glycemic control as shown in Fig. 2.

\section{CONCLUSION}

Periodontal therapy with oral azithromycin has effectively reduced HbA1c. Hence, periodontal therapy with oral azithromycin can be employed as a supportive strategy for management of diabetes mellitus. Henceforth, prevention and control of periodontal disease along with antibiotics must be considered an integral part of glycemic control. However, due to the lesser sample size in this study, further investigations are required to confirm the effect of periodontal therapy on systemic diseases.

\section{ACKNOWLEDGMENT}

The authors are thankful to the management of Vels University for providing excellent research guidance and support.

\section{REFERENCES}

1. Sreeja CN, Mable SJ, Anoop KR. Etiopathogenesis of chronic inflammatory periodontitis. J Pharm Pharm Sci 2012;4:43-9.

2. Marchetti E, Monaco A, Procaccini L, Mummolo S, Gatto R, Tete S, et al. Periodontal disease: The influence of metabolic syndrome. Nutr Metab (Lond) 2012;9:88

3. Loe H. Periodontal disease: The sixth complication of diabetes mellitus. Diabetes Care 1993;16:329-34.

4. Berezow AB, Darveau RP. Microbial shift and periodontitis. Periodontol 2000 2011;20055(1):36-47.

5. Kumar PS, Griffen AL, Barton JA, Paster BJ, Moeschberger ML, Leys EJ. New bacterial species associated with chronic periodontitis. J Dent Res 2003;82(5):338-44.

6. Negrato CA, Tarzia O, Jovanovic L, Chinellato LE. Periodontal disease and diabetes mellitus. J Appl Oral Sci 2013;21(1):1-12.

7. Shlossman M, Knowler WC, Pettitt DJ, Genco RJ. Type 2 diabetes mellitus and periodontal disease. J Am Dent Assoc 1990;121(4):532-6.

8. Fernando L, Santiago AH, Raul C. Relationship between diabetes and periodontal infection. World J Diabetes 2015;6:927-35.

9. Preshaw PM, Alba AL, Herrera D, Jepsen S, Konstantinidis A, Makrilakis K, et al. Periodontitis and diabetes: A two-way relationship. Diabetoloqia 2012;55(1):21-31.

10. Stanko P, Izakovicova HL. Bidirectional association between diabetes mellitus and inflammatory periodontal disease. A review. Biomed Pap Med Fac Univ Palacky Olomouc Czech Repub 2014;158(1):35-8.

11. Patil V, Mali R, Mali A. Systemic anti-microbial agents used in periodontal therapy. J Indian Soc Periodontol 2013;17(2):162-8.

12. Gladue RP, Bright GM, Isaacson RE, Newborg MF. In vitro and in vivo uptake of azithromycin (CP 62993) by phagocytic cells possible mechanism of delivery and release at sites of infection. Antimicrob Agents Chemother 1989;33(3):277-82.

13. Girard AE, Girard D, English AR, Gootz TD, Cimochowski CR, Faiella JA, et al. Pharamacokinetic and in vivo studies with azithromycin (CP-62,993), a new macrolide with extended half-life and excellent tissue distribution. Antimicrob Agents Chemother 1987;31(12):1948-54

14. Blandizzi C, Malizia T, Lupetti A, Pesce D, Gabriele M, Giuca MR, et al. Periodontal tissue disposition of azithromycin in patients affected by chronic inflammatory periodontal diseases. J Periodontol 1999;70(9):960-6

15. Arvind V, Jaiganesh R. Local drug delivery systems in the treatment of periodontitis - An overview. Int J Pharm Pharm Sci 2012;4(1):30-7.

16. Noyan U, Yilmaz S, Kuru B, Kadir T, Acar O, Buget E. A clinical and microbiological evaluation of systemic and local metronidazole delivery in adult periodontitis patients. J Clin Periodontol 1997;24(3):158-65.

17. Feier I, Onisei D, Onisei D. The plurivalence of the interpretation of correlation between plaque score and bleeding score. Int J Med Dent 2009;13(1):45-8

18. Dodwad V, Ahuja S, Kukreja BJ. Effect of locally delivered tetracycline hydrochloride as an adjunct to scaling and root planing on Hbalc, C-reactive protein, and lipid profile in type 2 diabetes: A clinicobiochemical study. Contemp Clin Dent 2012;3(2):150-4.

19. Shaddox LM, Walker CB. Treating chronic periodontitis: Current status, challenges, and future directions. Clin Cosmet Investig Dent 2010;2:79-91

20. Goodson JM. Antimicrobial strategies for treatment of periodontal diseases. Periodontol 2000 1994;5:142-68.

21. Dastoor SF, Travan S, Neiva RF, Rayburn LA, Giannobile WV, Wang HL. Effect of adjunctive systemic azithromycin with periodontal surgery in the treatment of chronic periodontitis in smokers: A pilot 
study. J Periodontol 2007:78(10):1887-96.

22. Lai PC, Ho W, Jain N, Walters JD. Azithromycin concentrations in blood and gingival crevicular fluid after systemic administration. J Periodontol 2011;82(11):1582-6.

23. Hanes PJ, Krishna R. Characteristics of inflammation common to both diabetes and periodontitis: Are predictive diagnosis and targeted preventive measures possible? EPMA J 2010;1(1):101-16.

24. Stewart JE, Wager KA, Friedlander AH, Zadeh HH. The effect of periodontal treatment on glycemic control in patients with type 2 diabetes mellitus. J Clin Periodontol 2001;28(4):306-10. 